\title{
Study of SUSY particles properties at the future International Linear Collider with the International Large Detector Concept
}

\section{Katarzyna Wichmann*}

DESY, Notkestr. 85, 22607 Hamburg, Germany

E-mail:kklimek@mail.desy.de

\begin{abstract}
Recently, Letters of Intent (LoI) for experiments at the International Linear Collider (ILC) have been submitted. Among the three proposals is the International Large Detector (ILD) concept for which a wide spectrum of studies of SUSY particle properties is presented here. Most of them are benchmark reactions for the ILC and can be used both in physics studies and in work on detector design and optimization, respectively. All studies were performed with a full detector simulation using GEANT4. The importance of this fully detailed simulation is reflected in the results. The presented analyzes have been chosen to be the most challenging for the detector to study its performance and guide the detector development. Additionally the problem of unavoidable beam induced backgrounds at linear colliders is addressed and ways of reducing its impact on physics studies are shown for an example SUSY analysis.
\end{abstract}

The 2009 Europhysics Conference on High Energy Physics,

July 16 - 222009

Krakow, Poland

* Speaker. 


\section{Introduction}

The International Linear Collider (ILC) is the next big project in particle physics. The ILC will collide electrons and positrons at energies of initially $500 \mathrm{GeV}$ with a possibility of an upgrade to $1 \mathrm{TeV}$. The ILC plans for an ambitious physics program, to extend and complement studies at the Large Hadron Collider (LHC). A characteristic feature of ILC physics is precision. Thanks to the clean initial state a lepton collider is a perfect machine for achieving very high precision. More details about the project and its physics case can be found elsewhere [1].

To be allowed to take full advantage of the physics potential of the ILC, the detector is required to exhibit an unprecedented performance. This is a challenge for the detector design and optimization, as well as for the reconstruction methods. Many detector parameters, for example vertex, tracking or jet energy resolutions, must be better than in existing experiments. The detailed requirements and resulting challenges for detectors at the ILC are described in the ILC RDR [1].

The International Large Detector (ILD) is a concept for a detector at the ILC. It is a multipurpose detector that provides excellent precision in spatial and energy measurement over a large solid angle, efficient reconstruction of secondary vertices and excellent momentum resolution for charged particles. The design combines excellent calorimetry and tracking to obtain the best possible overall event reconstruction, including the capability to reconstruct individual particles within jets, which is essential for particle flow calorimetry, favored as the ILC reconstruction method.

An significant problem faced by any linear collider are unavoidable beam induced backgrounds. A short introduction to beam background is presented here, as well as ways of reducing its impact on physics studies are shown for a sample SUSY analysis.

\section{SUSY at the Linear Collider}

The ILC community has defined a number of challenging benchmark physics reactions, which, to the best of the current knowledge, will form an important part of the physics program at the ILC. The benchmark reactions stress study of the Higgs boson and Supersymmetry (SUSY) as a model for a possible new physics scenario. They also probe the missing energy measurement capability of the detector, jet energy resolution and assume excellent lepton and flavor tagging. Most of the benchmark reactions can be used both in physics studies and in work on detector design and optimization. The presented analyzes have been chosen to be the most challenging for the detector to study its performance and guide the detector development.

All studies were performed with a full detector simulation using GEANT4, which is a great improvement compared to previous results with much less detailed simulation (SIMDET).

All studies were done for the $500 \mathrm{GeV}$ center-of-mass energy.

\subsection{Neutralino and Chargino Separation}

One of the benchmark processes for the optimization of the detector concepts proposed for the ILC is chargino and neutralino pair production in an mSugra scenario "Point5" [2], where the $\tilde{\chi}_{1}^{ \pm}$ and $\tilde{\chi}_{2}^{0}$ are mass degenerate and decay into $W^{ \pm} \tilde{\chi}_{1}^{0}$ and $Z^{0} \tilde{\chi}_{1}^{0}$, respectively. Especially interesting is the case with a fully hadronic decay mode of the vector bosons, where the final state consists of 4 jets and missing energy. Essential for the distinction between the two reactions is excellent $W / Z$ 

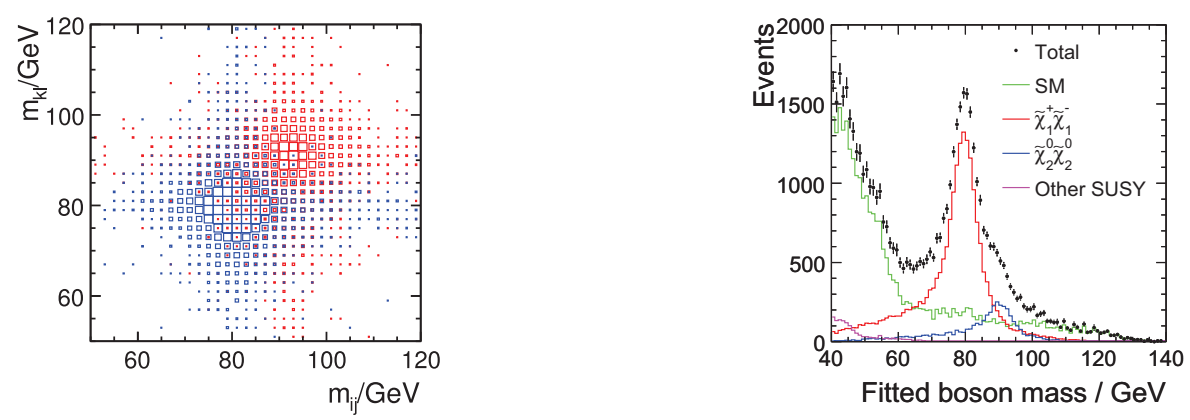

Figure 1: On the left: reconstructed di-jet mass distribution for $v \bar{v} W W$ (blue) and $v \bar{v} Z Z$ (red) events. On the right: reconstructed mass of vector boson candidates after all selection cuts.

separation. This separation is very sensitive to the jet energy resolution and thus to the particle flow performance.

Fig 1, left, presents an example di-jet mass distribution for $v \bar{v} W W$ and $v \bar{v} Z Z$ events, showing a very good separation of $W$ and $Z$ jets. Fig 1 , right, shows the reconstructed boson mass distribution obtained after all selection cuts which can be used to determine gaugino masses. The masses of gauginos can be obtained via the energy spectrum of the $W$ and $Z$ boson candidates, since the distribution of gauginos is box-like with edges determined by the masses and the center-of-mass energy. The sample must be separated on an event-by-events basis into $\tilde{\chi}_{1}^{ \pm}$and $\tilde{\chi}_{2}^{0}$ pair candidates, which is done using di-jet masses. The masses of $\tilde{\chi}_{1}^{ \pm}, \tilde{\chi}_{2}^{0}$ and $\tilde{\chi}_{1}^{0}$ can be determined with a statistical precision of $2.9 \mathrm{GeV}, 1.7 \mathrm{GeV}$ and $1.0 \mathrm{GeV}$, respectively.

\subsection{Long-lived Neutralinos}

For the optimization of the electromagnetic calorimeter (ECAL) a very challenging reaction is the reconstruction of highly energetic, non-pointing photons. This signature could appear from the decay of long-lived SUSY particles. Reconstruction of the mass and lifetime of the original SUSY particle requires a good photon energy reconstruction, as well as an excellent photon pointing resolution and thus is a good way to test the performance of the ECAL.

In the Gauge-Mediated SUSY Breaking (GMSB) scenario $\tilde{\chi}_{1}^{0}$ decays in to a gravitino and a photon. For some range of SUSY parameters $\tilde{\chi}_{1}^{0}$ might decay in the detector tracking volume. For this case a signal for the GMSB $\tilde{\chi}_{1}^{0}$ production is a pair of photons with a production point displaced from the origin. The signal is thus two non-pointing $\gamma_{\mathrm{s}}$. Non-pointing photons were reconstructed using a photon-finding algorithm based on cluster patterns of the ECAL cells [3]. The angular resolution drops from better then $1 \%$ for $90^{\circ}$ to $5 \%$ for $40^{\circ}$ incident angle in the ECAL barrel part. The excellent angular distribution of reconstructed photons in the ECAL allows the $\tilde{\chi}_{1}^{0}$ life time to be determined from the photon impact parameter distribution. For neutralino lifetimes of 0.2-2 ns a signal to background ratio of approximately unity can be achieved. This allows the neutralino mass to be determined with a precision of $\pm 2 \mathrm{GeV}$, the lifetime to be measured to $1 \%$.

\subsection{Stau Mass and Polarization}

For the SUSY SPS1a' parameter set, the process $e^{+} e^{-} \rightarrow \tilde{\tau} \tilde{\tau} \rightarrow \chi_{1} \tau \chi_{1} \tau$ gives in the final state missing energy and relatively low energy visible products of tau decays. This measurement 

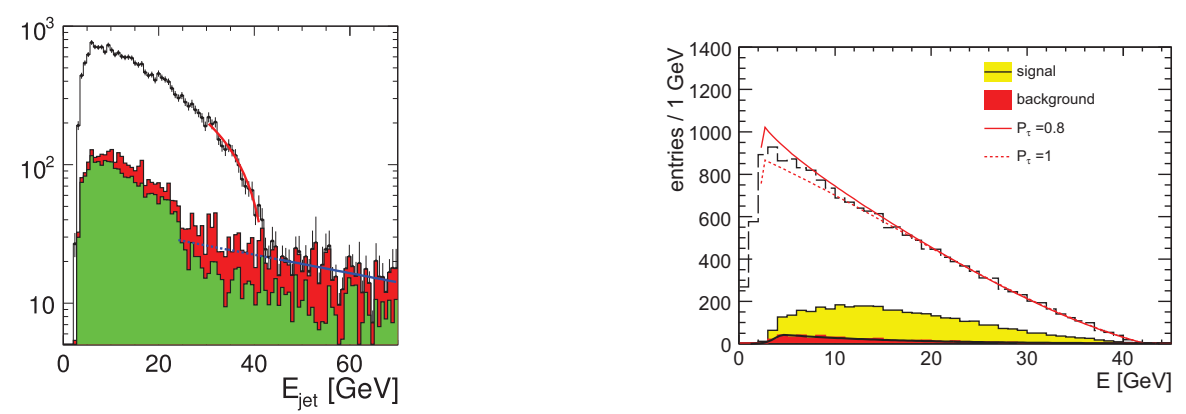

Figure 2: On the left: Tau-jet energy spectrum. Black: total spectrum; Red: total background; Green: SUSY background. Blue line: fit to background (the solid part is the fitted function, the dashed is it's extrapolation into the signal region); Read line: fit to total spectrum minus fitted background. On the right: Energy spectrum of selected tau decays and curves for different values of $P_{\tau}$.

requires precision tracking of relatively low momentum particles, good particle identification, high hermeticity of the detector, and low machine background.

The $\tilde{\tau}$ mass can be extracted from the end-point of the tau-jet energy spectrum, shown in Fig. 2, left, and the known $\tilde{\chi}_{1}^{0}$ mass [4]. The statistical uncertainty on the end-point is $0.1 \mathrm{GeV}$. Accounting for the uncertainty on the $\tilde{\chi}_{1}^{0}$ mass, $\sigma_{L S P}$, gives a precision on $M_{\tilde{\chi}_{1}^{0}}$ of $0.1 \mathrm{GeV} \oplus 1.3 \sigma_{L S P}$.

The measurement of tau polarization, $P_{\tau}$, in $\tilde{\tau}$ decays gives direct access to the mixing of mass and interaction eigenstates in the stau sector, and thus has sensitivity to a number of SUSY parameters. $P_{\tau}$ can be measured most cleanly in $\tau \rightarrow \pi v$ decays, where the slope of the $\pi^{ \pm}$energy spectrum depends on $P_{\tau}$. This measurement is very sensitive to the spectrum shape which strongly depends on the quality of particle identification. This fit sensitivity to the spectrum shape is illustrated in Fig. 2, right, which shows the reconstructed data together with curves for different values of $P_{\tau}$. This study shows that a measurement of $P_{\tau}$ with an accuracy of 0.15 is realistic.

\section{Beam Induced Backgrounds at the Linear Collider}

Lepton linear colliders face a novel problem - beam induced backgrounds. At the ILC machine induced backgrounds will be an important source of unwanted interactions [5]. The most prominent beam related background are so called "beamstrahlung" photons coming from beam-beam interactions and $e^{+} e^{-}$pairs produced by these photons. The photons are strongly focused in the forward direction and exit through the beam tube, mostly being of no consequence for the physics events at the interaction point. The electron-positron pairs however, can easily reach the detector, both the direct particles and the scattered ones. The machine and detector designs take into account possible ways of reducing the $e^{+} e^{-}$pairs spray reaching the detector but the remaining background needs to be taken into account on the reconstruction and analysis level.

The $e^{+} e^{-}$pairs can be simulated using Ginuea Pig generator [6]. The background studies were based on the expected simulated detector hits from a big pool of bunch crossing (BX) of these pairs. The hits were then overlayed on simulated physics events taking into account estimated readout rates of the ILD components.

Recently a big improvement has been made in the reconstruction packages that remove unwanted background hits in the Time Projection Chamber (TPC), which is the ILD main tracking 

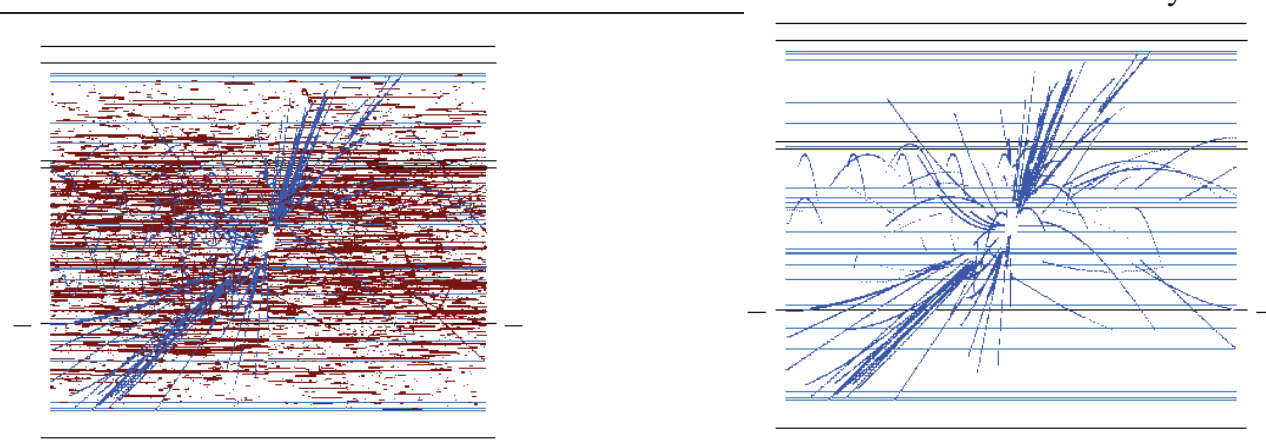

Figure 3: On the left: the $r-z$ view of the TPC hits from a $t \bar{t}$ event (blue) with 150 BXs of beam background (red) overlayed. On the right: the same event showing the reconstructed TPC tracks after the micro-curler removal algorithm applied and TPC track finding done.

detector. In this study $t \bar{t}$ events were overlayed with $150 \mathrm{BXs}$ of pair-background hits. On average there are 265000 background hits in the TPC, compared to about 23100 signal hits. A significant fraction of the background hits in the TPC comes from low energy $e^{+} / e^{-}$from photon conversion which form small-radius helices parallel to the z-axis ("micro-curlers"). Specific pattern recognition software has been written to identify and remove these hits prior to track reconstruction. The cuts remove approximately $99 \%$ of the background hits and only $3 \%$ of physics hits. Less than $1 \%$ of hits from tracks with $p_{T}>1 \mathrm{GeV}$ originating from the $t \bar{t}$ event are removed. Figure 3 shows that this level of background hits proves no problem for the track-finding pattern recognition software.

The SUSY study of SPS1a' stau mass, described in the previous section 2.3 has been repeated with a full beam background simulation added to the physics signal. Each physics event has been overlayed with $1 \mathrm{BX}$ of $e^{+} e^{-}$pairs. The SUSY reaction is characterized by a missing energy and two low multiplicity tau-jets in the final state and requires, additionally to good tracking, particle identification and hermeticity, low machine background. To remove extra tracks coming from the beam background topological cuts have been used. The cuts require that energy of reconstructed particles $E>0.5 \mathrm{GeV}$ and each particle has at least one hit in the TPC. After this additional selection the DELPHI jet algorithm has been used for tau-jets finding. It is more efficient in a presence of extra tracks and clusters from beam background than the standard Durham jet algorithm used in non-background analysis. The final results of this analysis showed that there is negligible difference in $\tau$ mass extracted with beam background and without.

\section{References}

[1] ILC Project, “International Linear Collider Reference Design Report 2007.” ILC-REPORT-2007-001

[2] J. List, T. Suehara, DESY-09-124, arXiv:0908.0876 [hep-ex]

[3] N. Wattimena and J. List, “Long-lived $\tilde{\chi}_{1}^{0}$ in Gauge Mediated SUSY Breaking”, ILD Analysis Note, 2009. http://www.ilcild.org/documents/ild-loi-material/neutralinoDecays.pdf/at_download/file

[4] P. Schade et al., "Measurement of $\tau$ polarisation in stau decays at the ILC". http:// www.ilcild.org/documents/ild-loi-material/LC-Note.pdf/at_download/file

[5] A. Vogel, “PhD thesis”, Universitaet Hamburg, 2008. DESY-THESIS-2008-036

[6] D. Schulte "PhD Thesis", DESY/Universitaet Hamburg, 1997. DESY-TESLA-97-08 